\title{
Editorial: ERMSAR-2017 conference of NUGENIA/SARNET on research on severe accidents in nuclear power plants
}

\author{
J ean-PierreVan Dorsselaere, SandroPaci
}

Despite accident prevention measures adopted in present nuclear power plants (NPP), some accidents, in circumstances of very low probability, may develop into severe accidents with core melting and plant damage and lead to the dispersal of radioactive materials into the external environment, thus constituting a hazard for the public health and for the environment. This risk was unfortunately underlined by the Fukushima-Daiichi accidents in J apan in March 2011.

The SARNET network of excellence (Severe Accident Research NETwork), coordinated by the Institut de Radioprotection et de Sûreté Nucléaire (IRSN, France), has been launched in 2004 and co-funded by the European Commission in the framework of the $6^{\text {th }}$ and $7^{\text {th }}$ Research and Development Framework Programmes (FP6 and 7) until 2013. The aim was to better coordinate the national efforts in Europe, optimising the use of the available expertise and of the experimental facilities, in view of resolving the remaining issues for enhancing the safety of existing and future European NPPs. This network was a success with the consolidation of the sustainable integration of the European severe accident research capacities and significant research achievements, also in collaboration with the main worldwide organization involved in the severe accident field. In mid-2013, the network was fully integrated in the NUGENIA European association (see www. nugenia.org) that addresses R\&D on Generation II and III NPPs. The SARNET activities have continued in the NUGENIA Technical Area N² (TA2) "Severe Accidents" framework, still coordinated by IRSN. This TA2 encompasses the former network activities but also extends them to the issues of "emergency and preparedness response" and "severe accident impact on the environment". The main TA2/ SARNET activities are focalized on the share of knowledge through specific technical workshops, the elaboration of new R\&D projects, the organization of periodic ERMSAR (European Review Meeting on Severe Accident Research) conferences, and the organization of education and training courses, as the recent one in October 2017, hosted by the J ožef Stefan Institute (J SI) in Ljubljana (Slovenia).

The $8^{\text {th }}$ ERMSAR conference, which selected papers are hosted in this Annals of Nuclear Energy special issue, was hosted by the Polish National Center for Nuclear Research (NBCJ) organization in Warsaw (Poland) from 16 to 18 May 2017. The Scientific Programme Committee involved eight researchers from diverse organizations (CEA, CIEMAT, ENEA, IRSN, J SI, KIT and University of Pisa). The conference gathered 179 participants from 27 countries and 84 organizations ( $25 \%$ of participants was from out of Europe such as J apan, Russia, USA, Singapore, China and Korea), confirming its status of a major international event on nuclear reactor severe accidents. Sixty-five papers and fourteen posters were presented during the Meeting and a significant time was allocated after each presentation for questions and open discussions.

During the plenary introductive session, after the NCBJ general presentation and the overview of NUGENIA/SARNET activities, two invited papers addressed respectively the Euratom research activities related to severe accidents within the HORIZON 2020 frame and the OECD-WGAMA R\&D activities. Three other invited papers presented respectively the FASTNET FP7 project on fast emergency tools, the IRSN approaches to the evaluation of the behaviour of radionuclides in continental hydro-systems next to a severe accident and, finally, the overview of the severe accident R\&D programme in South Korea.

Five main sessions were organized during ERMSAR 2017: in-vessel corium and debris behaviour, ex-vessel corium interactions and coolability, containment behaviour including hydrogen explosion risk, source term, and severe accident scenarios plus two poster sections on the same matters. All these topics included both experimental and modelling 
activities, as well as the assessment of the applicability of simulation codes to real plant scenarios. The main high-priority topics of the NUGENIA R\&D roadmap have been furthermore addressed. This special issue of Annals of Nuclear Energy journal includes a selection of 15 ERMSAR-2017 papers (all the papers presented during the Meeting are available as open documents on the SARNET website www.sar-net.eu), performed by the Scientific Programme Committee.

\section{Session "in-Vessel Corium and debris behaviour” (15 papers)}

The $R \& D$ in this area aims at reducing the remaining uncertainties on the possibility of cooling the reactor core structures and materials during a severe accident, either in the original core region or in the vessel lower head, so as to limit the progression of the accident. This goal could be achieved either by ensuring the retention and the cooling of the corium within the reactor vessel by a water injection, or at least a slow corium progression and small flow rates of the corium release into the containment cavity. These issues are linked to severe accident management (SAM) procedures for current reactors and also to the design and safety evaluations of future NPPs.

Among the 15 papers presented during this session that addressed the corium behaviour in the vessel lower head or the In-Vessel-Melt-Retention (IVMR), the following ones were selected:

- "Status of the IVMR project: First steps towards a new methodology to assess InVessel Retention Strategy for high-power reactors" by F. Fichot (IRSN) et al.,

- "Validation Progress and Exploratory Analyses of Three-Dimensional Simulation for BWR In-Vessel Core Degradation" by T. Okawa et al. (NRA),

- "Modeling issues related to molten pool behavior in case of In-Vessel Retention Strategy" by L. Carénini et al. (IRSN),

- "Experimental and Numerical Investigation of Molten Corium Behavior in Lower Head under External Subcooling and Boiling Conditions" by P. Pandazis (GRS) et al.,

- "On the use of CALPHAD-based enthalpy-temperature relations in suboxidized corium plane front solidification modelling" by R. Letellier et al. (CEA).

- Melting Test of Penetrating Tube through BWR-RPV Bottom Wall" by M. Pellegrini et al. (IAE)

The first one presents the relevant IVMR H2020 project, led by IRSN, which gathers most active organizations on the IVMR issue, both on theoretical and experimental aspects. The three following papers deal respectively: the 3D simulation of BWR core degradation phenomena with code validation on QUENCH, FARO and KROTOS experiments; modelling in the IRSN ASTEC integral code of the corium behaviour in the vessel lower head; and simulation of LIVE experiments in external vessel cooling conditions employing the ATHLETCD GRS code. The fifth paper concerns a general formulation of energy conservation equations in terms of specific enthalpies to account for the dependence of enthalpytemperature relations on the corium chemical composition while the last selected paper of this section presents an experiment on the melting of a BWR in-core monitor guide tube which penetrates the bottom wall of the vessel lower head, using a corium composed of $\mathrm{UO}_{2}, \mathrm{ZrO}_{2}$ and $\mathrm{Zr}$..

\section{Session "Ex-Vessel Corium interactions and coolability" (10 papers)}

The investigated situation, after a postulated failure of the vessel lower head, is the corium presence in a reactor cavity that is initially dry but with the possibility of a water injection later on during the molten-core-concrete-interaction $(\mathrm{MCCl})$. Furthermore, steam explosions may be caused by the fuel-coolant-interaction $(\mathrm{FCl})$ after the corium pouring into the flooded reactor cavity. 
Most of the presented papers addressed $\mathrm{FCl}$ and debris coolability. Among them, the 3 following papers were selected for the present special issue:

- "Experiment and Modeling of J et Breakup in Fuel-Coolant Interactions" by K.H. Bang (KMOU), on the analysis, employing the TRACER II simulation code, of the COLDJ ET experiment using the Woods metal as corium simulant,

- "Study on thermal fragmentation characteristics of a superheated alumina droplet" by R. Meignen (IRSN) and W. Ma (KTH) et al., on the analysis of MISTEE $\mathrm{KTH}$ experiments to assess the heat and mass transfer modelling of the coolant during the fragmentation process in the $\mathrm{FCl}$ code IRSN MC3D,

- "Improvement of molten jet fragmentation modeling in MAAP" by A. Le Belguet (EDF), on new model developments of heat transfer between melt and the surrounding environment and of evaluation of the size of debris particles resulting from the corium jet fragmentation, and finally validation vs. the FARO J RC/ Ispra tests.

The other papers presented at the Meeting were related on the corium spreading under water, the $\mathrm{MCCl}$ process and the fabrication of fuel debris simulating the Fukushima Daiichi real ones.

\section{Session "Containment behaviour including hydrogen explosion risk" (7 papers)}

The $R \& D$ focus for the papers presented in this section is on the threats to the containment integrity caused by highly energetic phenomena, particularly the steam explosion and the hydrogen combustion. Hydrogen combustion (deflagration and detonation) may be caused by the ignition of a gas mixture characterized by high local hydrogen concentrations, which may be due to the imperfect mixing of the containment internal atmosphere.

Diverse subjects were presented during this session: Computational Fluid Dynamics (CFD) code applications, experiments on spray performance, analysis of different THAI experiments, and behaviour of passive auto-catalytic recombiners.

The only selected paper, "Benchmark exercise $\mathrm{TH} 27$ on natural convection with steam injection and condensation inside the extended THAI facility" by M. Freitag (Becker Technologies) et al., shows the conclusions of an international code benchmark in the frame of the German THAl program. This benchmark on the TH-27 test addressed the phenomena of steam mixing, condensation and stratification behaviour of light gases under containment typical flow conditions. Thirteen participating organizations employed lumped-parameters or CFD codes that generally showed a high prediction quality.

\section{Session "Source Term" (9 papers)}

Most of the papers for this session focused on the mitigation of source term and on the fission product chemistry. The main emphasis was on iodine and ruthenium nuclides, given their high radio-toxicity and volatility.

The 2 selected papers for this journal's special issue address the mitigation of the source term:

- "Mitigation of source term in suppression pools: large uncertainties in predictability" by L.E. Herranz et al. (CIEMAT), on the analysis of current models of suppression pools, as a passive way to scrub radioactivity from the gas reaching aqueous volumes, and on their validation vs. few experiments. This paper highlights the need of new suitable models and of well-characterized low submergence new experiments.

- "Main results of the European PASSAM project on severe accident source term mitigation" by T. Albiol (IRSN) et al., on the final outcomes of a past FP7 Euratom project, coordinated by IRSN with 9 partners. This project, mainly of $R \& D$ 
experimental nature, studied existing systems (i.e. water scrubbing, sand bed filters plus metallic pre-filters) and innovative ones (i.e. high pressure sprays, electrostatic precipitators, advanced zeolites and combined wet-dry filtration systems) for enhancing the reduction of radioactive waste that may be released into the environment following the meltdown of the core. This led to a significant extension of the current experimental database on such systems.

\section{Session "Severe accident scenarios" (15 papers)}

Most papers presented during this section were about the prediction capabilities of computer integral codes (mainly ASTEC, MAAP and MELCOR) as well as the evaluation of severe accident management procedures. A majority focused on modelling of in-vessel phenomena and corium propagation in PWR or BWR. Note that some posters underlined the current big efforts in Asia to build and validate new integral codes (Korea, J apan and soon People's Republic of China).

Three papers have been selected for this session. The first two papers summarize the outcomes of important international projects that took place in the last years in Euratom frame:

"Severe accident code to code comparison for two accident scenarios in a spent fuel pool" by 0 . Coindreau (IRSN) et al., on the AIR-SFP benchmark exercise, carried out in the frame of the NUGENIA+ project, involving 14 organizations employing 6 different severe accident codes. One of the conclusions was about the applicability of severe accident codes, which were initially developed for reactor applications, to the analysis of transients in spent fuel pools, but highlighting the necessity of a careful examination of modelling assumptions, a deep understanding of the simulated processes and considering the high uncertainty of simulation results;

"CESAM - Code for European Severe Accident Management, EURATOM project on ASTEC improvement" by H. Nowack (GRS) et al. on the improvement of the ASTEC computer code to simulate NPP behaviour throughout accident sequences including SAM measures. This project, led by GRS, involved 19 partners. The work included ASTEC modelling improvements, its validation vs. relevant experiments, creation of reference input decks for all reactor types operated in Europe today, and plant applications with simulation of SAM measures in various NPP types.

The third selected paper, "Studies on the Recriticality Potential during Fukushima Unit-3 Core Reflooding" by P. Darnowski (WUT), presents an investigation of the potential for recriticality phenomenon occurrence during the early in-vessel phase of the FukushimaDaiichi Unit 3 accident. The criticality calculations were performed employing the SERPENT2 code, a Monte Carlo neutron transport solver, and based on the accident progression and the core state as results of preliminary MELCOR 2.1 code analysis.

\section{Conclusions and SARNET perspectives}

The SARNET network activities are continuing in NUGENIA frame. Indeed, the reorientation of these activities from pure knowledge-based $R \& D$ towards $R \& D$ for prevention and mitigation of severe accident consequences has been confirmed (as already underlined by on-going EURATOM projects that are linked to TA2/ SARNET). However, for the future, the usability of the obtained $R \& D$ results must be analysed and probably improved. About this aspect, one recommendation for the next ERMSAR conference was the request of a specific PSA2 session.

As final impression, it was good to see ERMSAR still fully active 12 years after the $1^{\text {st }}$ ERMSAR in 2005 in Aix-en-Provence. A large number of young researchers were present in Warsaw but also a number of participants that can be considered as "credited senior 
experts" (i.e. above 30 years' experience). It makes ERMSAR an excellent forum to share experience among researchers and engineers generations.

The next $9^{\text {th }}$ ERMSAR conference will be hosted by the UJV organization in Prague (Czech Republic) in spring 2019.

\section{Acknowledgements}

All the actors of the ERMSAR-2017 conference must be acknowledged, in particular the NCBJ host team (especially Maciej Skrzypek) and the Scientific Programme Committee: besides the guest editors below, Pascal Piluso from CEA (France), Luis Herranz from CIEMAT (Spain), Felice de Rosa from ENEA (Italy), François Bréchignac from IRSN (France), Ivo Kljenak from J SI (Slovenia) and Alexei Miassoedov from KIT (Germany). Last but not least, this special issue would not have been possible without the assistance of the Elsevier staff.

J ean-Pierre Van Dorsselaere (IRSN, France), TA2/ SARNET coordinator

Sandro Paci (University of Pisa, Italy), responsible for spreading of excellence activities in TA2/ SARNET 